\title{
Empleo de Explosivos en el Alivio de Tensiones Residuales de la Zona de Influencia Térmica de Uniones Soldadas
}

\author{
(Use of Explosives in the Reduction of Residual Stresses in the Heated Zone of Welded Unions)
}

\author{
J. L. Garcia-Jacomino ${ }^{1}$, J. Burgos Sola ${ }^{1}$, A.Cruz-Crespo ${ }^{1}$, M. Alvarez Luna ${ }^{1}$, J. Garcia Arteaga ${ }^{1}$. \\ ${ }^{1}$ Centro de Investigación de Soldadura (CIS), Facultad de Ingeniería Mecánica, Universidad Central "Marta Abreu” de Las Villas, \\ Carretera a Camajuani Km. 51/2, Santa Clara, Villa Clara, CP. 54830, Cuba \\ e-mail: jacomino@uclv.edu.cu
}

\begin{abstract}
Resumen
En el trabajo es estudiado el efecto del tratamiento con explosivos sobre las tensiones residuales de uniones soldadas de aceros al carbono. Se analizan dos modos de disposición de la carga explosiva (disposición lineal y disposición sinusoidal). Como resultado se obtiene que el tratamiento con explosivo por cualquiera de los dos métodos de colocación de carga ofrece un alivio de tensiones en el entorno de 50-60\%, demostrándose así la viabilidad del método para aumentar la fiabilidad de las uniones soldadas. Se concluye también que la disposición sinusoidal resulta más ventajosa al requerir de menor cantidad de carga explosiva.
\end{abstract}

Palabras clave: Tratamiento por explosivo. Alivio de tensiones residuales. Uniones Soldadas.

\begin{abstract}
In this paper, the effect of explosive charges as a method to reduce the residual stresses in the heated zone of welded unions was studied. A comparative analysis of two geometries of the explosive charge applied on welded unions was made: linear and sinusoidal. The comparative results obtained before and after the explosive treatment demonstrate its effectiveness by the reduction of the residual stresses. Residual stress reduction ranged in the order of 50 and $60 \%$, confirming that explosive treatment offers a viable solution to extend the life of the welded unions.
\end{abstract}

Keywords: Welded joint. Explosive treatment, Residual stress reduction.

\section{Introducción}

Como bien refiere un gran número de fuentes, entre los cuales Masubuchi y Mochizuki, la soldadura se caracteriza por la particularidad de los fenómenos de transferencia de calor de una fuente móvil, donde las dimensiones de la pieza llevan a variaciones desiguales de volumen por diferencia de gradientes de temperatura y a veces de coeficientes de dilatación lineal que bajo determinadas condiciones de embridamiento, generan mayor o menor grado de tensiones residuales [1] [2].

Las tensiones térmicas de compresión resultantes corresponderán a aquellas que producen una reducción de volumen equivalente a la acción de impedimento de la expansión. Cuando la máxima temperatura alcanzada no excede de cierto valor, dicho esfuerzo se ubica en el campo elástico y su valor es igual al múltiplo de la deformación especifica $(\Delta \mathrm{L} / \mathrm{Lo})$ por el modulo de elasticidad del material [2] [3].

En la Zona de Influencia Térmica (ZIT) de la unión soldada, donde se supera el valor de la temperatura de la zona elástica, las tensiones de compresión tienden a superar a las de fluencia a esa temperatura, pero en ningún momento lo logra. El

(Recebido em 22/06/2008; Texto Final em 16/03/2009). proceso inverso ocurre durante el enfriamiento en condiciones de movimiento restringido. Las zonas afectadas durante la deformación plástica en caliente resultan cortas para ocupar los nuevos espacios alcanzados y aparecen entonces, tensiones térmicas de tensión. Al alcanzarse la temperatura ambiente habrán quedado tensiones térmicas permanentes, cuyo valor es del orden del límite de fluencia del material [1] [2].

Las tensiones residuales pueden llegar a tener valores elevados no admisibles, por lo que es necesario reducir dichas tensiones, existiendo diferentes métodos que se agrupan en: tratamientos térmicos y tratamientos mecánicos, incluyendo precalentamiento, postcalentamiento, impactos, vibraciones, entre otros [2] [3] [4], que por sus particularidades, para el caso de construcciones o estructuras metálicas de gran porte para tratamientos "in situ" están limitados. Un método de tratamiento mecánico alternativo que funge como fuente de impacto localizado en la Zona de Influencia Térmica de una unión soldada de estructura voluminosa o de difícil acceso es la aplicación de carga explosiva [5] [6] [7] [8] [9]. Este método de alivio de tensiones no ha sido ampliamente difundido por requerir de un conocimiento específico en el área de uso de explosivos, por considerarse de alto riesgo y que exige medidas especiales de seguridad. No obstante, la simplicidad relativa del método, cuando se domina el uso adecuado de explosivos, hacen de ésta una variante atractiva. 


\section{Materiales y Métodos}

\subsection{Materiales empleados}

El material seleccionado para las probetas de 400x80x10 mm fue el acero de construcción ASTM A36, cuyas propiedades mecánicas son: límite de fluencia de $250 \mathrm{MPa}$ (36300 psi), límite de rotura de 400-550 MPa (58000 - 79800 psi) y 23\% de elongación en $50 \mathrm{~mm}$ de longitud calibrada [10].

El electrodo empleado en el trabajo de soldadura para la realización de las uniones fue el E6013 de 3 mm de diámetro, según calificación AWS [11].

\subsection{Soldadura de las probetas}

La sujeción de la probeta se realizó mediante un dispositivo de embridamiento para impedir la libre dilatación y contracción de la misma durante la aplicación del calor en el proceso de soldadura (Figura 1). El apriete de los tornillos fue realizado con una llave controlado a $130 \mathrm{~N} \cdot \mathrm{m}$, adecuando un apriete homogéneo en toda la sección de la probeta. Las chapas fueron colocadas con una separación de junta de $1.5 \mathrm{~mm}$ y la soldadura fue realizada con proceso por soldadura manual SMAW en posición plana y una corriente de $130 \mathrm{~A}$.

Durante la realización del proceso fueron registrados los valores de la corriente de soldadura, el voltaje de arco y el tiempo de realización del cordón. Los parámetros de corriente y voltaje de soldadura fueron medidos en tres puntos de la junta soldada, utilizándose la media de los mismos para los cálculos. Con el tiempo medido y la longitud del cordón fue determinada la velocidad de soldadura. Una vez terminada la soldadura, la probeta fue enfriada al aire hasta la temperatura ambiente para ser retirada luego, limpiando las escorias y salpicaduras presentes.

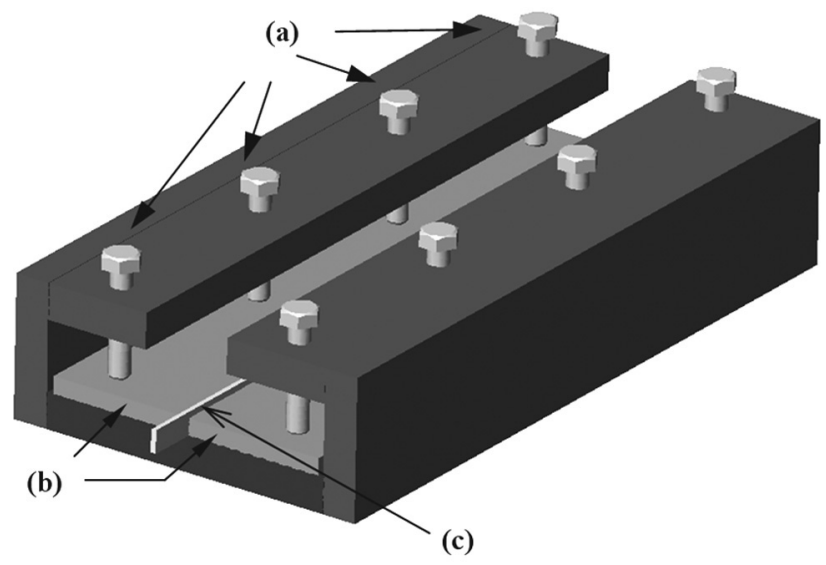

Figura 1. Dispositivo de sujeción: (a) tornillos de fijación, (b) probeta, (c) separador.

Las probetas fueron cortadas en dos secciones para someter una de las partes a tratamiento por explosión y en la otra medir el estado tensional postsoldadura.

\subsection{Cálculo de la Zona de Influencia Térmica o Zona Activa}

En la Zona de Influencia Térmica (ZIT) o Zona Activa $\left(B_{p l}\right)$ ocurren fenómenos de expansión contracción de la masa de metal fundido y del subyacente, el ancho va a estar determinada principalmente por los parámetros de soldadura que a la vez esta estrechamente relacionado por el tipo de proceso utilizado.

El cálculo de la zona activa $\left(B_{p l}\right)$ es igual a la suma de las dimensiones de la zona que tuvo una deformación plástica más la que tuvo una deformación elastoplástica. La primera ocurre donde el material alcanzó una temperatura aproximadamente igual a $600{ }^{\circ} \mathrm{C}$ y la otra donde hay una mezcla de deformaciones plásticas y elásticas.

El método utilizado en este trabajo fue el propuesto por Trochún [12], donde a partir de los valores de velocidad de soldadura $\left(\mathrm{V}_{\mathrm{s}}\right)$, Corriente de soldadura $\left(\mathrm{I}_{\mathrm{s}}\right)$, Voltaje de $\operatorname{arco}\left(\mathrm{V}_{\mathrm{a}}\right)$ y otros se calcula la Zona Activa $\left(B_{p}\right)$ con bastante exactitud conforme a la ecuación 1.

$$
B_{p l}=b_{1}+b_{2}
$$

Siendo:

$\mathrm{b}_{1}=$ ancho de la zona plástica, $\mathrm{cm}$

$\mathrm{b}_{2}=$ ancho de la zona elastoplástica, $\mathrm{cm}$

$$
b_{1}=\frac{0.484 * q_{o}}{C \rho * T^{*}}
$$

donde:

= energía lineal especifica de soldadura, $\mathrm{J} / \mathrm{cm}^{2}$

$C \rho=$ capacidad calorífica volumétrica, $\mathrm{J} / \mathrm{cm}^{3} \cdot{ }^{\circ} \mathrm{C}$

$T^{*}=$ temperatura de transición, ${ }^{\circ} \mathrm{C}$

$$
q_{o}=\frac{Q_{f}}{V_{s} * \delta_{o}}
$$

donde:

$Q_{f}=$ potencia de la fuente de calentamiento, $\mathrm{J} / \mathrm{s}$

$V_{s}=$ velocidad de soldadura, $\mathrm{cm} / \mathrm{s}$

$\delta_{o}^{s}=$ sumatoria de los espesores de las chapas a soldar,

$\mathrm{cm}$

$$
\begin{aligned}
& Q_{f}=V_{a} * I_{s} * \eta \\
& \text { donde: } \\
& V_{a}=\text { voltaje de arco, } \mathrm{V} \\
& I_{s}=\text { corriente de soldadura, A } \\
& \eta=\text { eficiencia del proceso de soldadura por arco } \\
& b_{2}=K_{2} *\left(H-b_{1}\right) \\
& \text { donde: } \\
& K_{2}=\text { coeficiente de forma empírico }[12] \\
& H=\text { espesor de la chapa a soldar, cm }
\end{aligned}
$$

En la Tabla 1 se muestran los valores de los parámetros de soldadura medidos durante la realización del experimento, así como los resultados de los cálculos de la Zona Activa $\left(B_{p l}\right)$. 
Tabla 1. Parámetros de Soldadura y Resultados del Cálculo de la Zona Activa $\left(B_{p l}\right)$.

\begin{tabular}{|c|c|c|c|c|c|c|}
\hline \multirow{2}{*}{$\begin{array}{l}\mathbb{E} \\
\stackrel{\mathbb{U}}{0} \\
0 \\
0 \\
0\end{array}$} & \multicolumn{3}{|c|}{$\begin{array}{c}\text { Parámetros de } \\
\text { Soldadura }\end{array}$} & $\begin{array}{c}\text { Ancho } \\
\text { zona plástica }\end{array}$ & $\begin{array}{l}\text { Ancho zona } \\
\text { elasto-plástica }\end{array}$ & Zona Activa \\
\hline & $\mathrm{I}_{\mathrm{s}}(\mathrm{A})$ & $\mathrm{V}_{\mathrm{a}}(\mathrm{V})$ & $\mathrm{V}_{\mathrm{s}}(\mathrm{cm} / \mathrm{s})$ & $b_{1}(\mathrm{~cm})$ & $b_{2}(\mathrm{~cm})$ & $B_{p l}(\mathrm{~cm})$ \\
\hline $\mathrm{A}, \mathrm{B}, \mathrm{C}, \mathrm{D}, \mathrm{E}$ y $\mathrm{F}$ & $128-131$ & $25-28$ & $0.18-0.25$ & $0.87-1.16$ & $1.57-1.63$ & $2.50-2.73$ \\
\hline
\end{tabular}

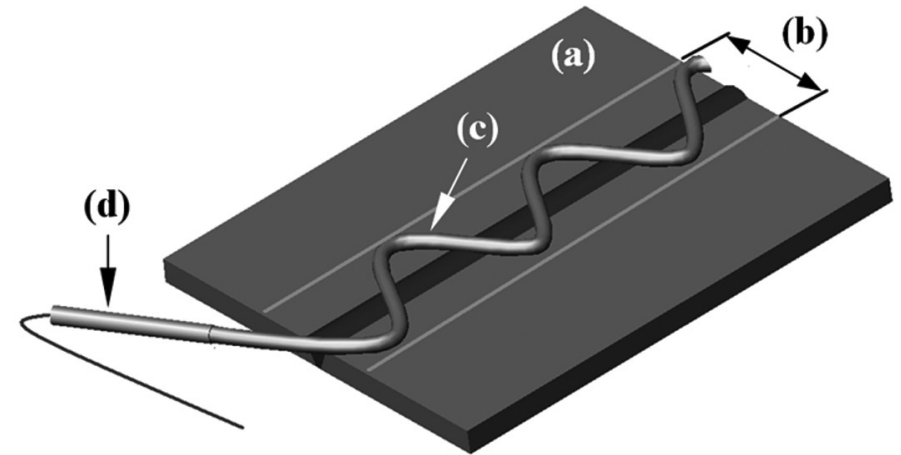

Figura 2. Disposición sinusoidal del cordón detonante: (a) probeta, (b) zona activa, (c) cordón detonante, (d) detonador.

\subsection{Reducción de tensiones residuales con explosivo}

Una vez realizado el cálculo de la zona activa se marcó la misma directamente en la chapa, utilizando un lápiz cristalográfico, de modo que permitiera identificar la zona donde se va a colocar el explosivo y que actúe directamente sobre la misma.

Existen diferentes formas de colocación del explosivo [1] [5] [14]; en este trabajo fueron utilizadas dos variantes de disposición para el cordón detonante:

$\checkmark$ Variante I: Disposición sinusoidal del cordón detonante sobre el cordón de soldadura (figura 2).

$\checkmark$ Variante II: Disposición de dos hilos paralelos del cordón detonante al cordón de soldadura (figura 3 ).

Fue empleada cinta adhesiva para mantener al cordón detonante en la posición correcta. El cordón detonante utilizado tiene 10,6 gramos de explosivo por metro, con núcleo de Hexógeno [15].

Durante la realización de toda la etapa experimental se cumplieron las medidas de seguridad establecidas para el trabajo con explosivos [16]. Las probetas se llevaron al polígono de pruebas, fueron situadas sobre apoyo plano en el lugar, con las condiciones requeridas para efectuar la detonación. Una vez realizada la detonación se aprecia que la superficie de la probeta no se daña por el efecto de la onda de choque, solo en el lugar de contacto entre el explosivo y la chapa existe una ligera huella,

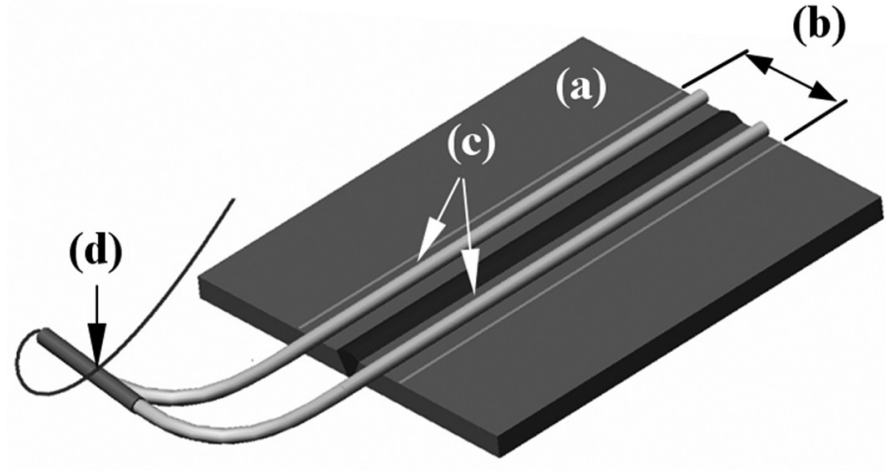

Figura 3. Disposición hilos paralelos del cordón detonante: (a) probeta, (b) zona activa, (c) cordón detonante, (d) detonador.

casi imperceptible visualmente.

\subsection{Medición de las tensiones residuales}

La medición de las tensiones residuales fue realizada mediante extensómetros, utilizando el puente de Wheastone como circuito fundamental para medir de forma directa el desequilibrio eléctrico que se produce tras la deformación de las galgas [17] [18] [19].

Debido a que el método de medición empleado es destructivo y que además el impacto de la onda de choque del explosivo destruye las galgas, fue necesario seccionar en dos partes la probeta original, la primera para la medición de las tensiones "antes de la explosión" y la segunda para la medición de las tensiones "después de la explosión". Esto afecta la distribución y magnitud original de las tensiones residuales en el centro de la probeta, por donde se corta en fresadora con disco de corte bajo condiciones de enfriamiento, pero permite valorar el efecto del tratamiento aplicado ya que se mantiene un alto nivel de tensiones.

Los extensómetros utilizados son de fabricación japonesa marca Kyowa, cuyas características principales se pueden observar en la tabla 2. Los extensómetros fueron adheridos en el centro de las probetas, paralelos al cordón de soldadura, separados a una distancia de $10 \mathrm{~mm}$ uno de otro (figura 4).

Tabla 2. Características de los extensómetros utilizados.

\begin{tabular}{|c|c|c|c|c|}
\hline $\begin{array}{c}3 \\
\frac{2}{0} \\
\frac{0}{0}\end{array}$ & Factor de Galga & Medidas de la Base & $\begin{array}{c}\text { Longitud de la } \\
\text { Galga }\end{array}$ & $\begin{array}{c}\text { Resistencia de la } \\
\text { Galga }\end{array}$ \\
\hline KFG-20-120-C1-11 & 2.1 & $28 \times 8 \mathrm{~mm}$ & $20 \mathrm{~mm}$ & $120 \Omega$ \\
\hline
\end{tabular}




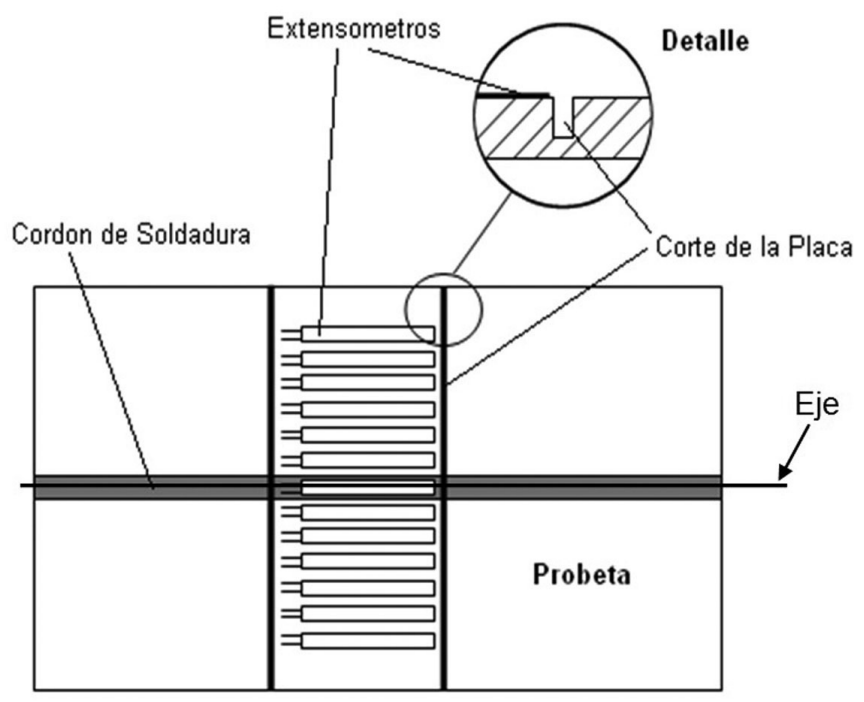

Figura 4. Colocación de los Extensómetros.

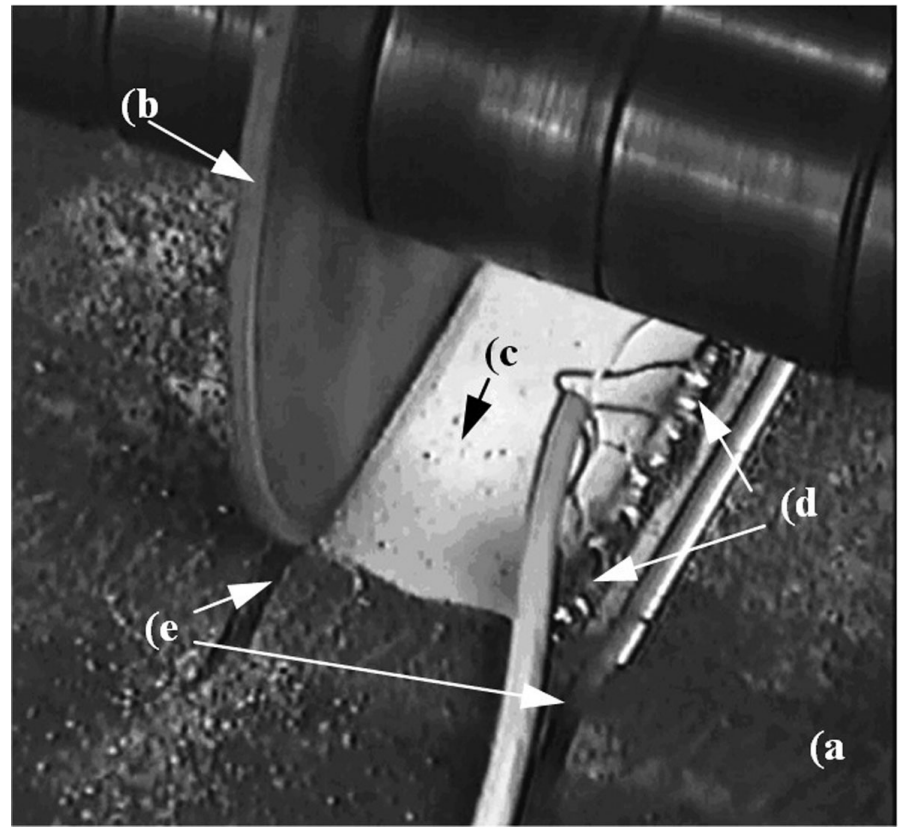

Figura 5. Corte de la probeta: (a) probeta, (b) disco de corte, (c) extensómetros cubiertos de parafina, (d), contactos eléctricos, (e) corte.

La preparación de la superficie de la probeta y la fijación de los extensómetros para efectuar las mediciones se hizo siguiendo los procedimientos establecidos para este fin [20]. Luego de tener los extensómetros adheridos a la superficie de la probeta se pegaron contactos eléctricos para evitar el deterioro de los sensores y se recubrieron con parafina. Se utilizó un conmutador a fin de medir la variación de voltaje de los extensómetros, con el objetivo de obtener los datos para el cálculo de las tensiones residuales longitudinales $\left(\sigma_{\mathrm{x}}\right)$.

El corte de las probetas para la medición de las tensiones residuales, según muestra la figura 5 , fue realizado con un disco de $1.5 \mathrm{~mm}$ de espesor, utilizando una fresadora horizontal 6P80. Fueron realizados dos cortes perpendiculares al cordón de soldadura y con velocidades mínimas para evitar la inducción de nuevos tensiones residuales o la alteración de los valores de tensiones ya existentes. Esta prueba fue efectuada con tres probetas ( 1 experimento y 2 réplicas) para cada tipo de disposición del cordón detonante.

\section{Análisis y discusión de los resultados}

Los resultados del cálculo de las tensiones residuales longitudinales $\left(\sigma_{x}\right)$ de las probetas antes de aplicarle el tratamiento con explosivos y después de hacerlo, en ambas variantes utilizadas de colocación del cordón detonante, se pueden observar en la Tabla 3 .

Los valores medidos de $\sigma_{\mathrm{x}}$ antes de aplicarle el tratamiento con explosivo, no muestran variaciones significativas, oscilando de un valor mínimo de $383.96 \mathrm{MPa}$ en la probeta A hasta un máximo de $418.29 \mathrm{MPa}$ en la probeta $\mathrm{D}$ sobre el cordón de soldadura, lo cual comprueba la homogeneidad de las condiciones en que se soldaron las probetas, y asegura que el dispositivo diseñado y construido para lograr el embridamiento necesario para el surgimiento de los tensiones residuales garantiza la fijación requerida de la probeta.

Las variaciones de los tensiones residuales antes y después de aplicarle explosivo demuestran que el método propuesto tiene efectividad. Por ejemplo, la probeta D muestra una tensión inicial de 418.29 $\mathrm{MPa}$ sobre el cordón de soldadura, al ser sometida al tratamiento, el esfuerzo disminuye hasta 223.37 $\mathrm{MPa}$, lo que representa el $46.6 \%$ con respecto a los tensiones residuales iniciales.

El resultado de esta variación se puede observar en la figura 6, la cual muestra la distribución promedio de los tensiones residuales longitudinales de las probetas en ambas variantes, que va desde un valor máximo de tensión en el centro del cordón de soldadura, luego comienza a disminuir a medida que se va separando del mismo, hasta pasar al campo de los tensiones residuales de compresión, siendo la suma total de estas tensiones igual a cero.

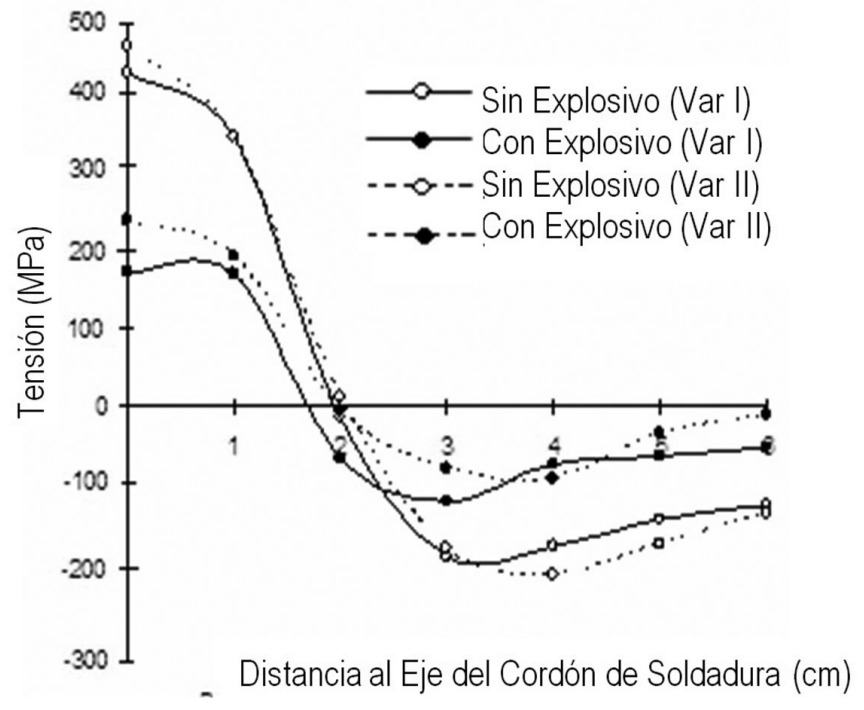

Figura 6. Distribución de los esfuerzos residuales. 
Tabla 3. Valores de las tensiones residuales longitudinales $\sigma_{\mathrm{x}}(\mathrm{MPa})$.

\begin{tabular}{|c|c|c|c|c|c|c|}
\hline \multirow{3}{*}{ Distancia (mm) } & \multicolumn{6}{|c|}{ Probetas en la Variante I de disposición sinusoidal del cordón detonante } \\
\hline & \multicolumn{2}{|c|}{ Probeta A } & \multicolumn{2}{|c|}{ Probeta B } & \multicolumn{2}{|c|}{ Probeta $\mathrm{C}$} \\
\hline & Antes & Después & Antes & Después & Antes & Después \\
\hline 0 & 383.96 & 177.55 & 372.48 & 163.22 & 406.83 & 166.08 \\
\hline 10 & 309.49 & 166.09 & 298.01 & 154.63 & 292.27 & 154.63 \\
\hline 20 & -28.62 & -45.79 & -22.89 & -45.79 & 28.62 & -40.07 \\
\hline 30 & -183.29 & -103.06 & -183.29 & -97.33 & -171.83 & -103.06 \\
\hline 40 & -177.56 & -68.70 & -171.83 & -62.97 & -200.49 & -62.97 \\
\hline 50 & -131.71 & -62.97 & -120.25 & -57.24 & -189.02 & -57.24 \\
\hline \multirow[t]{2}{*}{60} & -103.06 & -57.24 & -131.71 & -45.79 & -166.09 & -45.79 \\
\hline & \multicolumn{6}{|c|}{ Probetas en la Variante II de disposición dos hilos paralelos del cordón detonante } \\
\hline \multirow{2}{*}{ Distancia (mm) } & \multicolumn{2}{|c|}{ Probeta D } & \multicolumn{2}{|c|}{ Probeta E } & \multicolumn{2}{|c|}{ Probeta $\mathrm{F}$} \\
\hline & Antes & Después & Antes & Después & Antes & Después \\
\hline 0 & 418.29 & 223.37 & 401.1 & 209.03 & 395.43 & 220.5 \\
\hline 10 & 298.01 & 183.29 & 286.54 & 166.09 & 309.49 & 177.56 \\
\hline 20 & 40.07 & 11.45 & 22.89 & 5.72 & -28.62 & 5.73 \\
\hline 30 & -171.83 & -97.33 & -160.36 & -91.60 & -183.29 & -91.60 \\
\hline 40 & -200.49 & -68.70 & -206.22 & -80.15 & -200.49 & -80.15 \\
\hline 50 & -194.76 & -28.62 & -194.76 & -17.17 & -120.25 & -17.17 \\
\hline 60 & -177.56 & 5.74 & -183.29 & -5.72 & -103.06 & 0.00 \\
\hline
\end{tabular}

Nota: Los valores positivos corresponden a las tensiones residuales de tracción y los negativos a las de compresión

Distancia: con respecto al centro (eje) del Cordón de Soldadura.

Antes: se refiere a los valores antes de aplicar el tratamiento con explosivos.

Después: se refiere a los valores después de aplicar el tratamiento con explosivos.

El efecto de mejora de las propiedades de las uniones soldadas con explosivo se logra porque la onda de choque de compresión, que resulta de la detonación de la carga explosiva, colocada sobre la zona afectada térmicamente, provoca deformaciones plásticas locales del metal, llevando al recalcado en las capas superficiales y tensiones residuales de compresión. La acción de la carga explosiva cambia el campo de tensiones residuales en un volumen comparativamente grande de la unión soldada [13] [21] [22].

$\mathrm{Al}$ ocurrir la explosión de la carga en el material de la probeta a lo largo del cordón de soldadura surgen una serie de ondas de choque cilíndricas divergentes. Detrás del frente de cada una de estas ondas el material de la probeta comunica un movimiento desde la normal interior hacia la superficie del frente de la onda de choque. Las características de la forma de este movimiento tienen componentes que están dirigidos a lo largo del eje de la soldadura a ambos lados del eje de la carga. Por eso en las zonas de la probeta perpendiculares con relación al cordón de soldadura el material se alarga localmente a lo largo del eje de la soldadura, es decir, en dirección de las tensiones residuales de tracción longitudinales.

Las deformaciones de alargamiento de la soldadura y ZIT creadas por la explosión tienen, de esta manera, un carácter discreto. Estas deformaciones en suma con las deformaciones residuales elásticas homogéneas de alargamiento llevan a una deformación discreta irreversible de la soldadura y ZIT, gracias a lo cual se compensa el efecto de asentamiento de la soldadura, $\mathrm{y}$ consecuentemente, disminuyen las tensiones residuales.

Las dimensiones de la zona que sufre deformaciones de tracción dependen de la presión inicial en el frente de la onda de choque, o sea la potencia de la sustancia explosiva y además de las particularidades de su atenuación debido a los efectos de descarga y dispersión de la energía en el material deformado llevando a que las componentes longitudinales de la velocidad de masa detrás del frente de la onda de choque, así como la deformación determinada por el material, no sean iguales a diferentes distancias de la carga, y por esto la homogeneidad del tratamiento del material en su profundidad empeora a medida que la lamina se hace mas gruesa [21].

La diferencia entre una variante u otra de disposición de la carga explosiva es mínima; sin embargo, aplicar la variante I (300 $\mathrm{mm}$ de longitud de cordón detonante), tiene ventajas, ya que con menos cantidad de explosivos por metro lineal, se obtienen los mismos resultados que si se aplicara la variante II $(400 \mathrm{~mm}$ de longitud de cordón detonante), e inclusive ligeramente mejor. Esto se observa, por ejemplo, en la probeta $\mathrm{C}$ con disposición sinusoidal, de $406.83 \mathrm{MPa}$ disminuye a $166.08 \mathrm{MPa}$, lo que representa un $59.2 \%$ de atenuación de las tensiones residuales iniciales.

La disminución apreciada en las tensiones residuales luego 
de la aplicación del tratamiento con explosivo son sin dudas una evidencia de la disminución del riesgo de falla de las uniones soldadas, por tanto se puede asegurar que la fiabilidad de la misma aumenta y en correspondencia se eleva la integridad estructural de los elementos donde se aplica tal tratamiento de alivio de tensiones.

\section{Conclusiones}

El tratamiento con explosivo de uniones soldadas de acero es efectivo para disminuir las tensiones residuales de la Zona Afectada por el Calor, mostrando una variación $\Delta \sigma_{\mathrm{x}}$ de las tensiones en el entorno de 50-60\%, respecto al valor de tensiones residuales originadas por la soldadura. Ello significa evidentemente un aumento en la fiabilidad de la unión.

Entre las dos variantes utilizadas para la disposición de la carga explosiva la más efectiva y a su vez la más económica es la disposición sinusoidal del cordón detonante (Variante I), ya que con menor consumo de explosivos por metro lineal, se obtienen resultados similares (15.9 g para la Variante I y $21.2 \mathrm{~g}$ para la Variante II).

\section{Referencias}

[1] MASUBUCHI, K., Analysis of welded structures: Residual stresses, distortion, and their consequences. Massachusetts Institute of Technology, USA, pp.88-110, pp. 112-145, pp. 148187, (1980), pp. 328-335.

[2] MOCHIZUKI, M., et al., Fundamental study of thermal stress generation during welding heat cycles. Studies on numerical simulation of temperature, microstructure and thermal stress histories during welding, and their application to welded structures. Welding International. Vol. 19 (9), (2005), pp. 702710.

[3] KOU, S., Welding Metallurgy. A John Wiley \& Sons, Inc., Publication, (2002), pp. 400-415.

[4] CALISTER, W. D. Jr., Materials Science and Engineering and Introduction. A John Wiley \& Sons, Inc., Publication, (2000), pp. 328-350.

[5] PETUSHKOF, G., KUDINOF, B. M., TRUFIAKOF, B. Y., Parámetros de cargas de sustancias explosivas para eliminación de tensiones residuales soldadas a tope. Avtomatichescaia Esvarca. No 1, (1976), pp. 7-13.

[6] SCHMIDT, C. G. and SHOCKEY, D. A., Reducción de tensiones residuales en juntas soldadas con tratamientos con explosivo. Welding Journal, No 12, (1992), pp. 443-446.

[7] TRUFIAKOV, B. I., et al., Aumento de la resistencia al agotamiento de las uniones soldadas, con la utilización de cargas explosivas. Paton Welding Journal, No.5, (1976), pp. 32.

[8] DIDYK, R. P., et al., Explosive treatment as a means of redistribution of residual stresses in machine parts. Problemy Prochnosti. No. 11, (1994), pp. 50-54.

[9] PETUSHKOV, V.G., Peculiarities of explosion treatment of the circumferential weld on pipe filled with liquid. Paton Welding Journal. No.6, (2003), pp. 14-16.

[10] ASTM A36/A36M-04 Standard Specification for Carbon Structural Steel. West Conshohocken, PA.: American Society of Testing and Materials, (2004).

[11] ANSI/AWS A5.1-91, Specification for Carbon Steel

Electrodes for Shielded Metal Arc Welding. Miami, Fla.:

American Welding Society, (1991).

[12] FROLOV, V. V. , Teoría de los procesos de soldadura, Editorial Escuela Superior, Moscú, pp. 425-430.

[13] PETUSHKOV, V.G. and TITOV, V.A., Parameters of shock-wave loading used to relieve residual welding stresses by explosion treatment. Paton Welding Journal. No. 4, (2001), pp. 57.

[14] TITOV, V.A., Bryzgalin, A.G. and Petushkov, V.G., Limiting thickness of welded joints to be explosion treated. Paton Welding Journal. No.1, (2002) pp. 20-27.

[15] KOHLER, J. and RUDOLF, M., Explosives. 3rd edition, VHC Publishers, New York, (1987), pp. 83.

[16] NOM-005-STPS-1998, "Condiciones de Seguridad e Higiene en los Centros de Trabajo para el Manejo, Transporte y Almacenamiento de Sustancias Químicas Peligrosas" en Diario Oficial de la Federación. 15 de mayo de 1998. México, (1998).

[17] POBLET, M. J., et al, Transductores y medidores electrónicos. Revista Mundo Electrónico 2da edición (1983). No. 3, pp. 21-25.

[18] KANDIL, F.A., LORD, J.D., FRY, A.T., E GRANT, P.V., A Review of Residual Stress Measurement Methods - A Guide to Technique Selection, NPL Materials Centre, UK, (2001), pp 35-39.

[19] WITHERS, P. J., E BHADESHIA, H. K. D. H., Residual Stress - I: Measurement techniques. Materials Science and Technology. Vol. 17, (2001) pp. 355-365.

[20] GARCIA-JACOMINO, J.L. and R. QUINTANA-

PUCHOL, Procedimientos para la medición de tensiones mediante galgas extensométricas, in Centro de Investigaciones de Soldadura, Universidad Central "Marta Abreu" de Las Villas: Santa Clara. (2000) p. 23.

[21] JINXIANG, Z., et al., A study on the relief of residual stresses in weldments with explosive treatment. International Journal of Solids and Structures. Vol. 42 (13), (2005), pp 37943806.

[22] PETUSHKOV, V.G., Residual stresses in explosion treated pipes. Paton Welding Journal. No. 12, (2003), pp. 2-8. 